\title{
Tecnologías digitales innovadoras para el diseño de exoesqueletos para la mano aplicados en la rehabilitación de pacientes con artritis crónica
}

\author{
Roberto Moya-Jiménez ${ }^{a}$, Teresa Magal-Royo ${ }^{b}$, Diana Ponce \\ ${ }^{a}$ Universidad Central del Ecuador, Quito, Ecuador, rcmoya@uce.edu.ec, ${ }^{b}$ Universitat Politècnica de \\ Valencia, Spain,tmagal@degi.upv.es, 'Cuniversidad Central del Ecuador, Quito, Ecuador, \\ deponce@uce.edu.ec
}

\begin{abstract}
Resumen
El artículo describe la metodología planteada en el diseño de creación de un prototipo de exoesqueleto para la rehabilitación de la artritis reumatoide enfermedad inflamatoria, sistémica y crónica que afecta principalmente al sistema osteomuscular de pies, manos y que desemboca a la larga, en una discapacidad funcional severa.
\end{abstract}

La propuesta de diseño del exoesqueleto de la mano descrito en el artículo tiene en cuenta los accionamientos mecánicos aplicados sobre las articulaciones mediante el uso de servomotores conectados a hilos tensores que permiten el movimiento de flexo-extensión correcto de los dedos.

Se propone un planteamiento sistémico en la creación del prototipo final considerando los parámetros de esfuerzos y movimientos que se utilizan normalmente en la rehabilitación convencional y la creación de un dispositivo generado con técnicas de prototipado rápido de la mano tratada que permita diseñar un exoesqueleto único y exacto basado en las características antropométricas de cada paciente.

El resultado final ha permitido validar y verificar el proceso de desarrollo innovador de técnicas relacionadas con el Diseño Asistido por Ordenador, DAO y el Prototipado Rápido PR.

Palabras clave: Exoesqueleto, Innovación, Artritis Reumatoide, Prototipado rápido, Diseño Asistido por Ordenador. 
Tecnologías digitales innovadoras para el diseño de exoesqueletos para la mano aplicados en la rehabilitación de pacientes con artritis crónica

\section{Introducción}

La segunda área de mayor desarrollo tecnológico después del militar es el de la Sanidad, lo cual permite que la sociedad reciba ventajas considerables relacionados con la calidad y celeridad asistencial sobre todo en los procesos y la gestión adecuada de las tecnologías hospitalarias orientadas al usuario (Viaux, 2007).

La investigación sobre rehabilitación robótica de las extremidades superiores ha demostrado que la terapia con robots ayuda a recuperar la motricidad de la extremidad afectada (Prange et al, 2008), además puede mejorar los resultados funcionales (Kwakkel et al, 2008).

Los exoesqueletos como dispositivos tecnológicos avanzados consisten en un mecanismo estructural externo acoplado al ser humano de manera total o parcial que sirve como apoyo motriz, asistiendo los movimientos del usuario y aumentando sus capacidades motoras de manera global o parcial (Chávez et al, 2010).

Además, dentro de los exoesqueletos concebidos para la rehabilitación se debe considerar que deben incluir mecanismos elásticos poliarticulares capaces de generar fuerza muscular y ayudar activamente en la locomoción del paciente; a estos se los conoce como accionadores neumáticos, lo que permite reducir la carga biomecánica y muscular sobre el paciente (Renjewski \& Seyfarth, 2012).

El uso de actuadores para un exoesqueleto específicamente diseñado para las extremidades superiores como la mano, debe tener en cuenta la anatomía de la mano del paciente y que se cumplan con estándares de funcionalidad y usabilidad que se necesita para el control del exoesqueleto en función de las necesidades de tensión, extensión y compresión de los dedos y articulaciones de la mano. El Exoesqueleto utiliza sensores que estiman la fuerza producida por el humano y se encuentran incorporados en los actuadores de tipo SEA (Series Elastic Actuator) que se utilizan para amplificar la fuerza humana (Lopez et al. 2014). Además, mediante sensores se estima la posición y velocidad angular de las articulaciones, que se utilizan para controlar el movimiento.

Para la ayuda de personas discapacitadas como el dispositivo denominado HAL Hybrid Assistive Leg, (Kawamoto \& Sankai, 2002), desarrollado por la empresa Cyberdine que permite mejorar la debilidad muscular en pacientes mediante la amplificación de fuerza o como el dispositivo ReWalk (REHACARE, 2015), desarrollado por la empresa Argo Medical Technologies para parapléjicos y que funciona en base a trayectorias programadas.

Dentro de los ejemplos más relevantes del campo; Exo-Glove Ploy que puede ayudar a las personas con discapacidad de la mano, dispone de una banda palmar que conecta la parte de 
la mano y la parte dorsal de la parte portátil del dispositivo anclada a la muñeca, esta parte es rígida y se encuentra fija al paciente (Kang et al, 2012). El dispositivo NUS glove, es un guante que dispone de cuatro actuadores que se conectan son los dedos y que permiten realizar movimientos integrales de rehabilitación y asistencia en las actividades diarias, tales como el agarre y la extensión, es comúnmente recomendad en pacientes con padecimientos cerebrovasculares, derrame cerebral y artritis reumatoide (Yap et al, 2015). ETS-MARSE 2011 (Pons, 2008), Exoesqueleto de 7 GDL con capacidad terapéutica para hombro, codo, antebrazo y muñeca. Es controlado por torque y se encuentra montado en el piso. CADEN-7 2007 (Perry et al, 2007), Exoesqueleto de 7 GDL para la rehabilitación de hombro, antebrazo, codo y muñeca, se basa en un sistema de poleas. Power-Assist glove es un guante de asistencia desarrollado para mejora el movimiento usando una cubierta de plástico ubicada en los músculos que asisten el movimiento de flexión-extensión y el espiral de goma cubre el musculo para la asistencia del movimiento opuesto. La lámina de goma curvada y tela hacen que el guante sea compacto y flexible (Kadowaki et al,, 2011).

Siguiendo las líneas de investigación presentadas en los anteriores exoesqueletos, el presente artículo describirá el proceso de validación de prototipo de exoesqueleto compuesto por guante adaptado que produce compresiones de sujeción y extensión lo que permite al paciente con artritis mejorar los procesos de rehabilitación mediante ejercicios y con ello las funciones motoras de la mano.

\section{Metodología de desarrollo}

El estudio preliminar partió de la verificación de la eficacia del prototipo del exoesqueleto tomando en consideración los grados de libertad, GDL necesarios para el tratamiento de la enfermedad, el análisis mecánico de las cargas, los esfuerzos principales ejercidos, la resistencia en función de la forma y el material y finalmente el diseño formal del prototipo.

La rehabilitación se lleva a cabo a través de ejercicios terapéuticos. Según la APTA, American Physical Therapy Association (APTA, 2019) los ejercicios terapéuticos se definen como la aplicación sistemática planeada de movimientos físicos, posturas o actividades diseñadas para 1) remediar o prevenir deterioros, 2) mejorar el funcionamiento y 3$)$ mejorar la condición física.

El desarrollo del nuevo dispositivo se basa en criterios de rehabilitación, tomando en cuenta el diseño mecánico conceptual, la aplicación del dispositivo al paciente de prueba y factores de calidad que deben estar dispuestos a mejorar de acuerdo con las necesidades específicas del paciente en cuestión. 
Tecnologías digitales innovadoras para el diseño de exoesqueletos para la mano aplicados en la rehabilitación de pacientes con artritis crónica

El prototipo ajusta su funcionalidad mecánica GDL en los movimientos a realizar durante la rehabilitación de tipo activo y pasivo, en las cuales se incluyen rutinas de estabilización, control, movimiento. El proceso de creación del exoesqueleto comienza con la corrección de postura mediante la digitalización y comparación de las dimensiones antropométrica de la mano enferma con los patrones antropométricos de una mano tipo standard que puedan considerarse normales funcionalmente.

El sistema del prototipo fue verificado en un paciente con un estado inicial de la enfermedad donde la sintomatología presentada se asociaba a la pérdida de movimiento poliarticular en las manos, es decir cuando existen al menos cuatro articulaciones afectadas por la enfermedad y existe una disminución de la movilidad articular sin presencia de nódulos
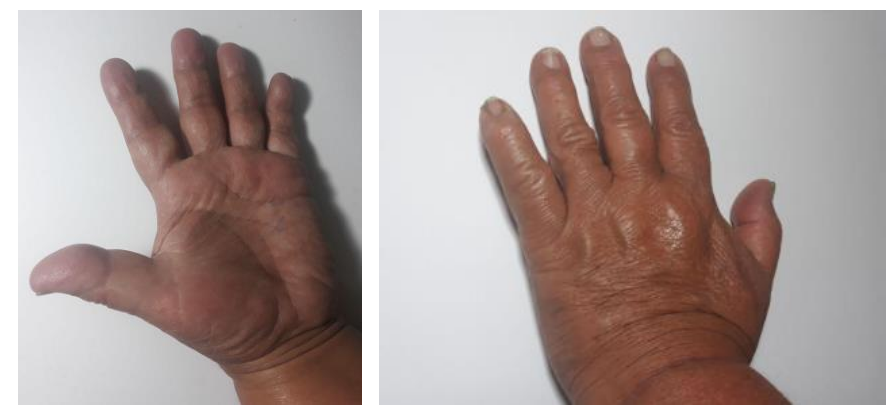

Fig. 1 Paciente con artritis reumatoide etapa inicial

\subsection{Prototipo Final.}

El prototipo consiste en una estructura de recubrimiento con orificios para la conexión de actuadores fijos a la mano del paciente mediante un guante, lo que permite desenvolver la acción de flexión y extensión con el uso de un servomotor que ejerce fuerza contraria para el movimiento.

Los mecanismos que envuelven cada dedo está conformado por unas guías que facilitan la transmisión del movimiento a las articulaciones dañadas. El tamaño y forma de estas piezas dependerán de la antropometría del paciente, teniendo en cuenta las deformaciones que la enfermedad haya ocasionado. El objetivo del dispositivo por tanto es lograr un movimiento orgánico de cada uno de los dedos ya que la estabilidad funcional del dispositivo dependerá de la sujeción de la estructura en la muñeca.

Se consideró no fijar las estructuras de los dedos a la base, debido a que actuadores deben encontrarse sincronizados a cada uno de los dedos en el que se efectuará el movimiento, y es imprescindible un rango de libre movimiento. 


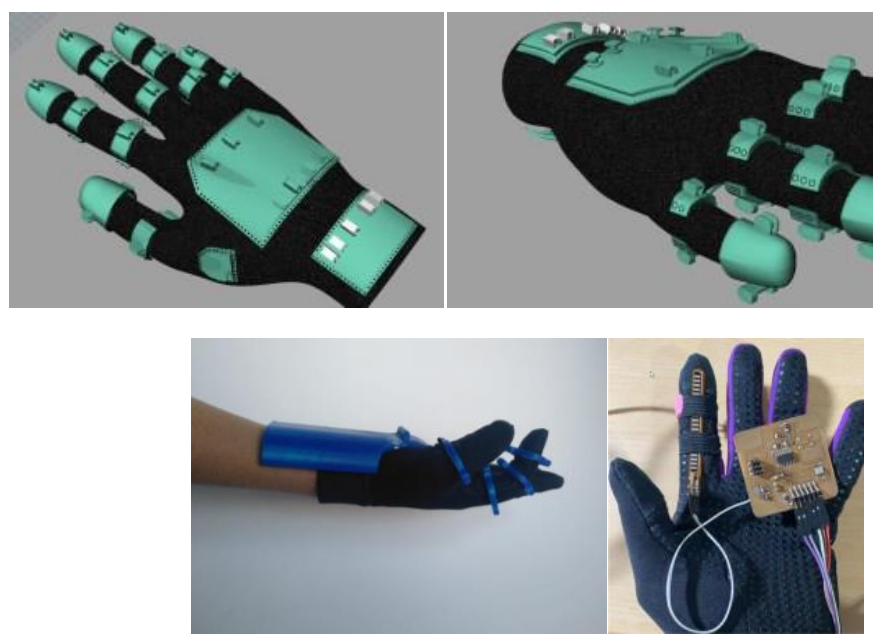

Fig. 2 Prototipo exoesqueleto.

Fuente: Creación propia.

En las terapias de rehabilitación para la mano se debe cumplir bajo normas internacionales, razón por la cual el dispositivo debe cumplir la normativa APTA antes mencionada. Se alcanzará un máximo de $58^{\circ}$ de amplitud de rango de movimiento en las articulaciones MCP, metacarpofalángica y en un ángulo aproximado de $60^{\circ}$ en las PIP, interfalángica proximal.

\section{Discusión}

En base a las investigaciones realizadas y a la obtención del prototipo final testeado en un paciente, se espera que el futuro puedan diseñarse pruebas a mayor escala en pacientes con necesidades de rehabilitación de la mano. Ello permitirá evaluar tantos los factores relacionados con la interacción del dispositivo-paciente como el ajuste de control del sistema de esfuerzos programados para cada paciente que permita detectar la mejora de la movilidad mediante una rehabilitación controlada terapeuticamente.

La obtención de estos datos determinará los avances futuros que se desarrollen sobre tecnologías medicas que permitan brindar una mejor calidad de vida al paciente e independizar la rehabilitación en enfermedades motoras. 
Tecnologías digitales innovadoras para el diseño de exoesqueletos para la mano aplicados en la rehabilitación de pacientes con artritis crónica

\section{Agradecimientos}

Esta investigación ha sido desarrollada gracias a los trabajos realizados durante la tesis doctoral "Estudio sistémico de estructuras exoesqueléticas de la mano para la rehabilitación de la atrofia muscular debido a la artritis en personas adultas" desarrollado en la Universitat Politécnica de Valencia.

\section{Referencias}

APTA. (2019). American Physical Therapy Association. https://www.apta.org/i Recuperado el 13 de 06 de 2020.

Chávez, \& et al. (2010). Exoesqueletos para potenciar las capacidades humanas y apoyar la rehabilitación. Colombia: Revista Ingeniería Biomédica, Vol.4(7), 63-73.

Kadowaki, Y., Noritsugu, T., Takaiwa, M., Sasaki, D., \& Kato, M. (2011). Development of Soft Power-Assist Glove and Control Based on Human Intent. Journal of Robotics and Mechatronics Vol.23(2), 281-291. DOI: 10.20965/jrm.2011.p0281

Kang, B. B., In, H., \& Cho, K. (2012). Force transmission in joint-less tendon driven wearable robotic Hand. In Proceedings 12th International Conference on Control, Automation and Systems (ICCAS), 1853-1858.

Kawamoto, H., \& Sankai, Y. (2002). Confortable power assist control method for walking aid by HAL-3. IEEE International Conference on Systems, Man and Cybernetics Vol.4 (6). DOI: 10.1109/ICSMC.2002.1173328.

Kwakkel, G., Kollen, B. J., \& Krebs, H. I. (2008). Effects of robot assisted therapy on upper limb recovery after. Neurorehabilitaion and Neural Repair Vol. 22(2) DOI: $10.1177 / 1545968307305457$.

Lopez, R., Aguilar, H., Salazar, S., Lozano, R., \& Torres, J. A. (2014). Modelado y Control de un Exoesqueleto para la Rehabilitacion de Extremidad Inferior con dos grados de libertad. Revista Iberoamericana de Automática e Informática industrial Vol. 11(3), 304-314. DOI:10.1016/j.riai.2014.02.008.

Perry, J. C., Rosen, J., \& Burns, S. (2007). Upper-Limb Powered Exoskeleton Design, in IEEE/ASME Transactions on Mechatronics, Vol. 12 (49), 408-417, DOI: 10.1109/TMECH.2007.901934.

Pons, J. L. (2008). Whearable Robots: Biomechatronic Exoesqueleton . Chichester, England: Jhon Whiley \& Sons. ISBN: 978-0-470-51294-4.

Prange, G., Jannick, M., Groothuis-Oudshoorn, H., \& Ijzerman, M. (2008). Systematic review of the effect of robot-aided therapy on recovery of the hemiparetic arm after stroke. Journal of Rehabilitation Research and Development. Vol. 43(2), 171-184, DOI:10.1682/jrrd.2005.04.0076. 
REHACARE Magazine. ReWalk Personal Exoskeleton System cleared by FDA for home use.https://www.rehacare.com/en/Archive/Archive_search/ReWalk_Personal_Exoskeleton_Syste m_cleared_by_FDA_for_home_use. Recuperado el 2 junio de 2020

Renjewski, D., \& Seyfarth, A. (2012). Robots in human biomechanics- a study on ankle push-off in walking. Bioinspiration \& Biomimetics, Vol.7(3) DOI: 10.1088/1748-3182/7/3/036005.

Viaux C.G.G. (2007) Penetración de las Tecnologías de la Información en las Realidades Hospitalarias de los Países Latinoamericanos y del Caribe. In: Müller-Karger C., Wong S., La Cruz A. (eds). IV Latin American Congress on Biomedical Engineering, Vol. 18. DOI: 10.1007/978-3-540-74471-9_263.

Yap, H. K., Goh, J. C., \& Yeow, R. C. (2015). Design and Characterization of Soft Actuator for Hand Rehabilitation Application. In Proceedings of the 6th European Conference of the International Federation for Medical and Biological Engineering FMBE Proceedings. DOI:10.1007/978-3-31911128-5_92. 